\title{
Estrategia didáctica basada en TIC para mejorar el desempeño académico en el área de Matemática
}

\section{ICT-based didactic strategy to improve academic performance in the area of Mathematics}

\author{
Doris Elizabeth Semanate-Semanate \\ doris.e.semanate.s@pucesa.edu.ec \\ Pontificia Universidad Católica del Ecuador, Ambato \\ Ecuador \\ https://orcid.org/0000-0003-4167-6878 \\ Darío Javier Robayo-Jácome \\ drobayo@pucesa.edu.ec \\ Pontificia Universidad Católica del Ecuador, Ambato \\ Ecuador \\ https://orcid.org/0000-0002-0661-6573
}

Recepción: 10 de mayo 2021

Revisado: 15 de junio 2021

Aprobación: 15 de agosto 2021

Publicación: 01 de septiembre de 2021 


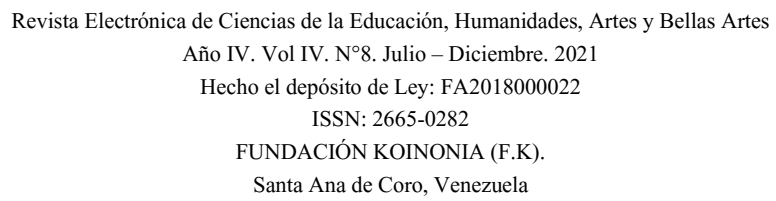

\title{
RESUMEN
}

Se realizó una investigación con el objetivo de desarrollar una estrategia didáctica basada en las TIC para la mejora del desempeño académico en el área de Matemática en los estudiantes de Segundo Año de Bachillerato de la Unidad Educativa Vicente León de Ecuador. Metodológicamente el estudio se desarrolló desde un enfoque cuantitativo, con un diseño cuasiexperimental y la aplicación de un pretest, una estrategia de intervención y un postest a una muestra de 60 estudiantes. Con los resultados se tiene que la estrategia didáctica basada en las TIC benefició la apropiación del conocimiento de la matemática, la capacidad de razonamiento crítico, la curiosidad intelectual, el mejoramiento cognitivo, la adquisición de contenidos a través de la teoría, la práctica y la experiencia; metodología que incentiva a los estudiantes en la responsabilidad, siendo parte activa en el entorno virtual.

Descriptores: Tecnología de la información; investigación pedagógica; modelo educacional. (Palabras tomadas del Tesauro UNESCO).

\begin{abstract}
An investigation was carried out with the objective of developing a didactic strategy based on ICT for the improvement of academic performance in the area of Mathematics in students of the Second Year of Baccalaureate of the Vicente León Educational Unit of Ecuador. Methodologically, the study was developed from a quantitative approach, with a quasi-experimental design and the application of a pre-test, an intervention strategy and a post-test to a sample of 60 students. With the results, it is clear that the ICT-based didactic strategy benefited the appropriation of the knowledge of mathematics, the capacity for critical reasoning, intellectual curiosity, cognitive improvement, the acquisition of content through theory, practice and learning. experience; methodology that encourages students in responsibility, being an active part in the virtual environment.
\end{abstract}

Descriptors: Information technology; educational research; educational models. (Words taken from the UNESCO Thesaurus). 


\section{INTRODUCCIÓN}

La sociedad actual demanda de los individuos el desarrollo de competencias desde sus capacidades intrínsecas para su autoaprendizaje, hasta las habilidades para comunicarse de manera efectiva y colaborativa. En este tenor, (García \& Benítez, 2011), proponen un conjunto de competencias relacionadas con los procesos intrínsecos de cualquier campo del saber: destrezas en la realización de análisis y síntesis, aprender; aplicar el conocimiento, resolver problemas; manejar tecnologías digitales; utilizar adecuadamente la información y trabajar con autonomía y en equipo.

De allí que, la educación tiene como propósito principal la formación integral del individuo con un pensamiento lógico, crítico y creativo que, indudablemente, le permita enfrentarse a los cambios continuos que vive la humanidad (Tamayo, Oscar, Zona, Loaiza, \& Yasaldez, 2015). En este sentido, hace falta generar innovación en el proceso de aprendizaje para desarrollar en el educando la capacidad de aprender a aprender de una manera eficaz y autónoma, tomando en cuenta sus propias necesidades con el fin de aplicar el conocimiento en los diversos contextos.

Considerando lo anterior, es menester resaltar que la Educación Matemática, en las últimas décadas ha sido objeto de investigación por su gran significancia; pues, como plantean (Martínez \& Pérez, 2016), la matemática en particular es una herramienta fundamental para interpretar y predecir las dinámicas y controles en la toma de decisiones que permite enfrentar grandes desafíos en distintos escenarios, sobre todo los económicos. De allí que se pueda apuntar que la competencia matemática, como afirma (Íñiguez, 2015), es la habilidad que tiene el estudiante para comprender, juzgar, crear y usar las matemáticas en distintos contextos intra y extra matemáticos.

No obstante, para ello se requiere dejar de lado las metodologías tradicionales y asumir la utilización de nuevas estrategias para poder enfrentar el reto de resolver problemas reales, con razonamiento, pensamiento y argumentación. Pues, son muchos los docentes que emplean metodologías sustentadas en la repetición de contenidos teóricos, aspecto que dificulta en la resolución de problemas matemáticos; además, del excesivo uso del 
dictado de la materia para que el estudiante copie en su cuaderno, lo que genera que estos asuman un rol pasivo, que solo recibe información (Fuentes, Paéz, \& Prieto, 2019). Para ilustrar, en el contexto ecuatoriano el proceso de enseñanza aprendizaje de la asignatura de matemática ha sido un reto para los docentes, considerando que los estudiantes del país han participado en diferentes pruebas nacionales (Ser Estudiante y Ser Bachiller) e internacionales como el Programme for International Student Assessment (PISA). Específicamente los resultados de la prueba Ser Estudiante (2017-2018) y Ser Baciller (2017-2018) no han sido del todo favorable, pues el campo de la matemática es el que presenta mayor dificultad para los estudiantes, dada la incapacidad exteriorizada de resolver problemas matemáticos (Heredia, 2021), tal como se expone a continuación:

\section{Tabla 1.}

Niveles de Logro en la Prueba Ser Estudiante/Ser Bachiller - Campo de la Matemática.

\begin{tabular}{|c|c|c|c|c|c|}
\hline \multirow{7}{*}{ 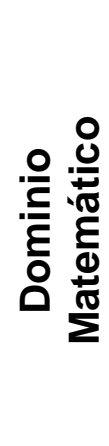 } & \multicolumn{5}{|c|}{ PRUEBA SER ESTUDIANTE } \\
\hline & $\begin{array}{c}\text { Grados } \\
\text { Evaluados }\end{array}$ & Insuficiente & Elemental & Satisfactorio & Excelente \\
\hline & $10^{\circ} E G B$ & $57,6 \%$ & $27,0 \%$ & $11,5 \%$ & $3,9 \%$ \\
\hline & $7^{\circ} E G B$ & $52,6 \%$ & $28,4 \%$ & $15,6 \%$ & $3,4 \%$ \\
\hline & $4^{\circ} E G B$ & $49,7 \%$ & $29,4 \%$ & $20,6 \%$ & $0,3 \%$ \\
\hline & \multicolumn{5}{|c|}{ PRUEBA SER BACHILLER } \\
\hline & $3^{\circ} \mathrm{BGU}$ & 27,5 & 43,7 & 25,9 & 2,9 \\
\hline
\end{tabular}

Fuente: Instituto Nacional de Evaluación Educativa, 2017-2018.

Ante la situación planteada, es menester asumir lo indicado por (Niebles, Hernández, \& Cardona, 2016) respecto a que en el sistema educativo actual es necesario que los maestros y maestras innoven y actualicen sus formas de enseñanza a través de la implementación de estrategias didácticas basadas en las tecnologías de la información y comunicación (TIC) que por su aspecto innovador y creativo beneficia la enseñanza y el 
aprendizaje de las matemáticas. Pues, este tipo de recursos ofrece a los estudiantes experiencias y situaciones que contribuyen con el desarrollo del pensamiento lógico y creativo mediante el planteamiento de posibles escenarios y actividades que incluyen la observación, la atención, la imaginación e intuición. Elementos que aportan al aprendizaje cooperativo y a la adquisición de competencias para su desarrollo personal, social y posteriormente profesional.

Hecha las observaciones anteriores, es oportuno considerar algunos argumentos teóricos y explicaciones que justifican y recalcan la importancia del uso de las TIC como estrategia que permite el fortalecimiento del desempeño académico en general y en el área de matemática en específico. En tal sentido, (Domínguez \& Carmona, 2017) sostienen que la introducción de las TIC en la vida de los seres humanos ha generado un cambio notable respecto a las formas de comunicar e informar, así, en el ámbito educativo contribuye a mejorar la manera de enseñar y aprender. Pues, desde esta perspectiva se logra un aprendizaje significativo al integrar el conocimiento, la teoría, la práctica y la experiencia. En este mismo tenor, (Khan \& Markauskaite, 2017) enfatizan en la importancia de la utilización de las TIC en el área educativa, al permitir la transferencia de conocimientos, el auto aprendizaje y la comprensión. Lo que quiere decir que las estrategias didácticas, los recursos tecnológicos y herramientas visuales, facilitan y mejoran la enseñanza y el aprendizaje, pues enriquecen los procesos que conllevan a la construcción del conocimiento.

Para (Faúndez, Bravo, Ramírez, \& Astudillo, 2017), la incorporación de la tecnología revela cambios en el proceso de formación y el rendimiento académico, dado por la adquisición de habilidades y actitudes que contribuyen en mejorar las capacidades mentales, el pensamiento crítico, el razonamiento lógico, el diálogo y la comunicación. Generándose de esta manera una constante innovación dentro de las aulas escolares al tener una ampliación de entornos de aprendizaje y diversificación de los métodos de trabajo. 
Cabe resaltar que el avance tecnológico beneficia el proceso de enseñanza aprendizaje al permitir el uso de estrategias didácticas tecnológicas para mejorar el desempeño académico de los distintos sujetos y en las diferentes áreas del conocimiento. De esta forma, como enuncian (Cardozo, Duarte, \& Fernández, 2018), se refuerzan las habilidades, las destrezas, valores y la racionalización del tiempo; su aplicación en el aula incrementa la motivación e interés en los educandos para resolver problemas en el contexto. Asimismo, el docente con su debida capacitación, se torna eficiente, productivo, eficaz, facilitador, orientador y guía; pues, se ve fortalecido en sus competencias y en la responsabilidad y compromiso con una educación con calidad y calidez.

En lo que respecta al diseño y ejecución de estrategias didácticas mediante el uso de tecnologías (Urgilez \& Valdez, 2020) afirman que estas se constituyen en componentes principales que benefician el ámbito formativo al contribuir en el modo de actuar, pensar y desarrollarse de los sujetos; además, facilitan el proceso de enseñanza y aprendizaje tanto de los educadores como de los educandos, mediante el acceso a la información, proceso de datos, expresión y comunicación; componentes generadores de experiencias desde la expresión escrita, gráfica y audiovisual. En otras palabras, la red se transforma en una herramienta de intercambio de información accesible y rápida que potencia el intercambio comunicativo y la creación de conocimientos.

En el ámbito específico de las matemáticas, (Herrada \& Baños, 2018) afirman que las estrategias didácticas basadas en las TIC es un aspecto estudiado en el área pedagógica que incluye la enseñanza de la matemática, resultando interesante e importante su aplicación para fortalecer el desempeño académico y reducir los elevados índices de fracaso escolar; además, en el proceso de aprendizaje se facilita la adquisición de competencias, la interacción, el diálogo y la comunicación; propicia el desarrollo de la iniciativa, la toma de nuevas decisiones, el trabajo autónomo, riguroso y metódico. No obstante, para que los profesores puedan utilizar la tecnología como recurso didáctico en el aula de clases y, así, facilitar el aprendizaje y la comprensión de los contenidos matemáticos, es indispensable cumplir lineamientos pedagógicos, metodológicos y 
tecnológicos (Albañil \& Tique, 2015). En tal sentido, es menester la constante actualización para el manejo de recursos tecnológicos (Herrera, Novelo, Díaz, \& Hernández, 2016), pues, todos los conocimientos y habilidades que tanto estudiantes como docentes puedan adquirir durante el proceso formativo, se verán potenciados si fortalecen el buen uso de las tecnologías y la creación de entornos de aprendizaje mediante el internet (Mainato \& Drouet, 2017).

Cabe agregar que todo lo planteado, conlleva a considerar a (Grisales, 2018), cuando afirma que el uso de la tecnología en la enseñanza de las matemáticas es un aspecto necesario y cuya aplicación se ha de sustentar en principios didáctico, metodológico y pedagógico. Argumentos tales, le dan contexto al desarrollo de un proceso de investigación a partir de cuestionar sobre las estrategias didácticas basadas en las TIC en el ámbito de las matemáticas en la Unidad Educativa Vicente León del Ecuador, considerando que su aplicación mejora del desempeño académico en el área de Matemática.

Finalmente, con la investigación se pretende desarrollar una estrategia didáctica basada TIC para la mejora del desempeño académico en el área de Matemática en los estudiantes de Segundo Año de Bachillerato de la Unidad Educativa Vicente León. Dado que, las estrategias basadas en las TIC ofrecen la oportunidad de ensayar nuevas respuestas o formas de actuar para superar conflictos académicos e impactar de manera significativa en el desarrollo cognitivo, procedimental y actitudinal como acciones que favorecen la formación integral de los educandos; además, de fortalecer la comunicación entre profesores y estudiantes mediante el uso de diversos recursos tecnológicos, que benefician la productividad y eficiencia del conocimiento. 
EPISTEME KOINONIA

Revista Electrónica de Ciencias de la Educación, Humanidades, Artes y Bellas Artes
Año IV. Vol IV. N ${ }^{\circ}$. Julio - Diciembre. 2021
Hecho el depósito de Ley: FA2018000022
ISSN: 2665-0282
FUNDACIÓN KOINONIA (F.K).
Santa Ana de Coro, Venezuela

Doris Elizabeth Semanate-Semanate; Darío Javier Robayo-Jácome

\section{METODOLOGÍA}

En este apartado se aclara la metodología asumida con la investigación. En tal sentido y considerando a (Palella y Martins, 2015), el estudio se fundamentó desde un enfoque cuantitativo con un diseño cuasiexperimental como estrategia para responder al problema de investigación. De igual manera, la investigación se caracterizó por desarrollarse con la aplicación de un pretest y postest con dos grupos de estudio similares, un grupo control y grupo experimental, al cual se le aplicó la estrategia didáctica basada en las TIC para la mejora del desempeño académico en el área de Matemática.

Con respecto a la muestra de la investigación es de saber que esta es una parte representativa de la población de estudio y de la cual se obtiene información (Palella y Martins, 2015). Entonces, la muestra estuvo conformada por un total de 60 estudiantes, en el rango de los 15 a 17 años, que cursan el Segundo Año de Bachillerato. Por consiguiente, 30 estudiantes integraron el grupo control y 30 estudiantes el grupo experimental.

Para recabar la información de los estudiantes se empleó la técnica de la encuesta. Para ello se utilizó como instrumento el cuestionario propuesto por Mejía, Toala, \& Valverde (2017) para evaluar el uso de la tecnología para el aprendizaje y la adquisición del conocimiento.

Con el objetivo de recabar información real con un error mínimo, se realizó la selección de interrogantes, al mismo tiempo se efectuó una adaptación al área de matemática. Tal cuestionario se estructuró mediante la Escala de Likert, la cual permite medir actitudes y conocer el grado de conformidad del encuestado y se aplicó a los estudiantes del área de matemática a través de la plataforma de Google Forms.

Dicha escala tiene la numeración de 1 a 5 para cada cuestión planteada: totalmente en desacuerdo (TDA $=1$ ), en desacuerdo $(E D=2$ ), ni de acuerdo ni en desacuerdo (NDA $\mathrm{NED}=3$ ), de acuerdo ( $\mathrm{DA}=4$ ), y totalmente de acuerdo ( $\mathrm{TA}=5$ ); el instrumento adquiere un nivel de confianza del 99\% (Matas, 2018). 
Después de aplicado el pre test para evaluar el uso de la tecnología en el desempeño académico se unifica los valores totalmente en desacuerdo y en desacuerdo criterios negativos, ni de acuerdo ni en desacuerdo neutro, de acuerdo y totalmente de acuerdo opinión positiva.

En lo que concierne al análisis de fiabilidad que proporciona información sobre las relaciones entre los elementos individuales de la escala, se tomó como referencia los resultados obtenidos en la eliminación por lista basada en todas las variables del procedimiento, obteniendo un total de 60 casos equivalente al $100 \%$, tal como se muestra a continuación.

\section{Table 2.}

Resumen del procesamiento de los casos.

\begin{tabular}{llcc}
\hline & & $\mathrm{N}$ & $\%$ \\
\hline \multirow{3}{*}{ Casos } & Válidos & 60 & 100,0 \\
\cline { 2 - 4 } & Excluidos $^{\mathrm{a}}$ & 0 &, 0 \\
\cline { 2 - 4 } & Total & 60 & 100,0 \\
\hline
\end{tabular}

Elaboración: Los autores.

Con respecto al nivel de fiabilidad y validez, cabe decir que estos se determinaron mediante el Programa Estadístico SPSS. En tal sentido, obtenida la información numérica y estadística a través de dicho programa, se estableció el coeficiente de Alfa de Cronbach que describe el nivel de consistencia interna basada en la correlación entre elementos. Los resultados arrojan una fiabilidad del 0,954 con 12 elementos que alcanza un criterio de excelente por su acercamiento a 1, tal como se presenta en el siguiente cuadro. 


\section{Tabla 1.}

Estadísticos de fiabilidad.

\begin{tabular}{cc}
\hline Alfa de Cronbach & N de elementos \\
\hline, 954 & 12 \\
\hline
\end{tabular}

Fuente: Test aplicado a los estudiantes.

\section{RESULTADOS}

A continuación, se presentan los resultados obtenidos tato en el pretest como en el postest, luego de aplicar la estrategia didáctica basada en las TIC para la mejora del desempeño académico en el área de Matemática en los estudiantes de Segundo Año de Bachillerato de la Unidad Educativa Vicente León. En tal sentido se presenta los siguientes resultados del pre test:

\section{Tabla 4.}

Instrumento (Pre Test) aplicado a los educandos.

\begin{tabular}{|c|c|c|c|c|c|c|}
\hline \multirow[b]{3}{*}{ N. } & \multicolumn{6}{|c|}{ Test para evaluar el uso de la tecnología en el desempeño académico en el área de matemática } \\
\hline & \multirow{2}{*}{ Preguntas } & \multicolumn{4}{|c|}{ Alternativas } & \multirow[b]{2}{*}{$\begin{array}{l}\text { TDA } \\
(5)\end{array}$} \\
\hline & & $\begin{array}{l}\text { TED } \\
(1)\end{array}$ & $\begin{array}{l}\text { ED } \\
(2)\end{array}$ & $\begin{array}{l}\text { NDA NED } \\
\text { (3) }\end{array}$ & $\begin{array}{l}\text { DA } \\
(4)\end{array}$ & \\
\hline \multicolumn{7}{|c|}{ Enseñar a pensar y razonar con el apoyo de recursos didácticos tecnológicos } \\
\hline 1 & $\begin{array}{l}\text { ¿Considera usted que la } \\
\text { utilización de recursos digitales } \\
\text { o enlaces virtuales mejora la } \\
\text { enseñanza de contenidos en } \\
\text { matemática? }\end{array}$ & 6 & 14 & 0 & 6 & 4 \\
\hline 2 & $\begin{array}{l}\text { ¿Los docentes en el área de } \\
\text { matemática promueven la } \\
\text { simulación, observación y } \\
\text { exploración en un ambiente } \\
\text { computacional? }\end{array}$ & 0 & 19 & 5 & 3 & 3 \\
\hline 3 & $\begin{array}{l}\text { ¿En el aula, se utiliza } \\
\text { herramientas tecnológicas } \\
\text { para enseñar conceptos, y } \\
\text { ejercicios matemáticos de } \\
\text { forma visual e interactiva? }\end{array}$ & 5 & 11 & 8 & 3 & 3 \\
\hline
\end{tabular}




\begin{tabular}{|c|c|c|c|c|c|c|}
\hline & \multicolumn{6}{|c|}{ Aprender a plantear y resolver problemas con la ayuda de herramientas virtuales } \\
\hline 4 & $\begin{array}{l}\text { ¿En el aula, las herramientas } \\
\text { tecnológicas que maneja el } \\
\text { docente ayuda en la búsqueda } \\
\text { de información y resolución de } \\
\text { problemas matemáticos? }\end{array}$ & 0 & 19 & 5 & 3 & 3 \\
\hline 5 & $\begin{array}{llr}\text { ¿Considera usted que las } \\
\text { tecnologías facilitan } & \text { la } \\
\text { realización de actividades y } \\
\text { tareas en el área de } \\
\text { matemática? }\end{array}$ & 0 & 19 & 4 & 4 & 3 \\
\hline \multirow[t]{2}{*}{6} & $\begin{array}{l}\text { ¿Cree usted que las } \\
\text { tecnologías manejadas por los } \\
\text { docentes del área de } \\
\text { matemática potencian un } \\
\text { aprendizaje activo, } \\
\text { participativo y creativo? }\end{array}$ & 0 & 19 & 5 & 3 & 3 \\
\hline & \multicolumn{6}{|c|}{ Construir modelos matemáticos con el uso de las tecnologías de la información y comunicaciór } \\
\hline 7 & $\begin{array}{l}\text { ¿Las TIC aplicadas por el } \\
\text { docente en el aula facilitan el } \\
\text { aprendizaje de cálculo mental y } \\
\text { escrito? }\end{array}$ & 0 & 19 & 5 & 3 & 3 \\
\hline 8 & $\begin{array}{l}\text { ¿Los recursos didácticos } \\
\text { tecnológicos empleados por } \\
\text { los maestros de matemática } \\
\text { promueven el razonamiento y } \\
\text { potencian su capacidad } \\
\text { intelectual? }\end{array}$ & 0 & 19 & 5 & 3 & 3 \\
\hline 9 & $\begin{array}{l}\text { ¿Las estrategias tecnológicas } \\
\text { empleadas en la enseñanza de } \\
\text { la matemática motivan, } \\
\text { estimulan la autonomía, la } \\
\text { autorreflexión y la participación } \\
\text { de los estudiantes? }\end{array}$ & 0 & 19 & 5 & 3 & 3 \\
\hline & Emplear recursos y herramienta & oló & & & & \\
\hline 10 & $\begin{array}{l}\text { ¿La tecnología utilizada por los } \\
\text { maestros de matemáticas en el } \\
\text { aula permite la visualización y } \\
\text { comprensión de relaciones } \\
\text { existentes entre los conceptos } \\
\text { y los objetos matemáticos? }\end{array}$ & 3 & 16 & 5 & 3 & 3 \\
\hline 11 & $\begin{array}{l}\text { ¿Usted mediante el uso de } \\
\text { internet favorece en el } \\
\text { intercambio de saberes y } \\
\text { despierta su interés por la } \\
\text { investigación en el área de } \\
\text { matemática? }\end{array}$ & 3 & 17 & 4 & 3 & 3 \\
\hline 12 & $\begin{array}{l}\text { ¿El docente utiliza recursos } \\
\text { tecnológicos (textos, vídeos, } \\
\text { archivos y audio visuales) que } \\
\text { facilitan el proceso de } \\
\text { enseñanza y aprendizaje, } \\
\text { siendo las clases atractivas y } \\
\text { dinámicas? }\end{array}$ & 0 & 22 & 2 & 3 & 3 \\
\hline
\end{tabular}


Fuente: Encuesta aplicada a los estudiantes.

Según los resultados obtenidos con la aplicación del pre test a los 30 estudiantes que integraron el grupo control y 30 estudiantes el grupo experimental se establece:

\section{En la dimensión enseñar a pensar y razonar con el apoyo de recursos didácticos} tecnológicos: el 67\% considera que los educadores no utilizan estrategias digitales o enlaces virtuales para la enseñanza de contenidos, el $63 \%$ no efectúa prácticas que beneficien en la exploración del ambiente computacional, el 53\% de maestros no emplea herramientas interactivas; aspectos que perjudican en la incorporación pedagógica de las TIC, generando barreras en el proceso de aprendizaje, y la adquisición de conocimientos mediante la interactividad e interconexión.

\section{En la dimensión aprender a plantear y resolver problemas con la ayuda de} herramientas virtuales: el $63 \%$ de estudiantes exterioriza dificultades en la búsqueda de información y solución de problemas matemáticos; únicamente, el 20\% afirma que las tecnologías empeladas por los maestros facilitan la realización de actividades y tareas en el área de matemática, al respecto el 64\% expone que no; Por tanto, en el aula la insuficiente utilización de diferentes aplicaciones ha limitado en la enseñanza a través del diseño de esquemas de trabajo estructurado, programas de gestión y materiales que permitan integrar de forma coherente códigos de información como texto, imagen, animación y sonido.

En la dimensión construir modelos matemáticos con el uso de las tecnologías de la información y comunicación: el 54\% expone que los docentes no potencian un aprendizaje activo, participativo y creativo, además, un $63 \%$ de estudiantes presentan dificultades en el cálculo mental y escrito; en igual porcentaje se resalta que los educadores demuestran desinterés en el uso de prácticas que promuevan el razonamiento y la capacidad intelectual para resolver problemas cotidianos. 
En la dimensión emplear recursos y herramientas tecnológicas: el $63 \%$ de maestros en el aula en ocasiones permiten la visualización y comprensión de relaciones existentes entre los conceptos y los objetos; el $67 \%$ de docentes no utiliza el internet, un porcentaje elevado del $70 \%$ de educadores no emplea redes de ordenadores electrónicos, (vídeos, archivos y audio visuales); además, los maestros exterioriza dificultades en el empleo de estrategias interactivas que motiven y estimulen en la autonomía, la autorreflexión y la participación de los estudiantes.

Según los resultados obtenidos se deduce que existe la necesidad fomentar el uso de estrategia didáctica fundada en las TIC que mejore el desempeño académico de los estudiantes en el área de matemática y, así, fomentar el conocimiento que conlleva a desarrollar niveles de seguridad para realizar procedimientos y confianza en la obtención de resultados, con una disposición consciente y favorable que promueve el emprendimiento de acciones para la solución de problemas. Pues, contrariamente, como expresan Cenich, Araujo, \& Santos (2020) la insuficiente utilización de estrategias didácticas tecnológicas afecta en el campo educativo; sin embargo, la adopción de una cultura tecnológica exige una reestructuración de los métodos de enseñar y aprender, de esta forma se disminuyen las prácticas tradicionales donde el conocimiento es visto como información transmitida y asimilada de forma pasiva en el proceso colectivo.

\section{Estrategia Tecnológica Matematic Flipped Classroom}

Tomando como referente los resultados obtenidos con la aplicación del pre test a los estudiantes en referencia al uso de las prácticas de enseñanza-aprendizaje y con la finalidad de disminuir las dificultades detectadas, se considera trascendental el diseño de una estrategia didáctica basada en las TIC.

En tal sentido, se presenta la siguiente propuesta de intervención dirigida a los estudiantes del Segundo Año de Bachillerato de la Unidad Educativa Vicente León con el propósito de mejorar el desempeño académico en la enseñanza- aprendizaje sobre el 
contenido matemático denominado Cónicas. Es de saber, que se asume la metodología de Flipped Classroom con un enfoque centrado en el constructivismo donde el educando fortalece la experiencia mediante la integración del conocimiento teórico y la práctica extraescolar.

\section{Descripción de la Estrategia Tecnológica "Matematic Flipped Classroom"}

La estrategia tecnológica "Matematic Flipped Classroom" se presenta en cinco secciones y el procedimiento de acceso se efectúa a través de la dirección web: https://sites.google.com/view/flippedclassroomconicas.

Presentación. Incluye una breve descripción del sitio Web con la finalidad de dar a conocer la importancia en el uso de la herramienta tecnológica.

Planificación. En este apartado, se detalla los elementos de la planificación como: objetivos de aprendizaje, nombre del proyecto, valores, contenidos, destrezas con criterio de desempeño, indicadores de evaluación y orientaciones metodológicas. La finalidad es cumplir con los lineamientos propuestos en el Currículo de Bachillerato Unificado, de esta forma se fortalece el perfil propuesto para los educandos en el nivel superior.

Secciones Cónicas. La sección consta de cuatro subpáginas referentes a las Cónicas: circunferencia, parábola, elipse e hipérbola. Cada una incluye cuatro etapas desarrolladas mediante la aplicación de la metodología de Flipped Classroom a través de actividades sincrónicas y asincrónicas.

- En la primera etapa se detalla las tareas extraescolares que deberán efectuarse mediante el uso de diferentes herramientas digitales como: YouTube, Canva, Genially.

- La segunda fase contiene tareas de reflexión que requiere el manejo de Google Drive que admite el almacenamiento de documentos en Word, 
aspecto que beneficia en el trabajo interactivo y colaborativo entre el educador y los educandos; es significativo destacar que la creación de organizadores gráficos se efectúa mediante la herramienta Mindomo, Padlet, Popplet y Coggle, herramientas que según (Ministerio de Educación del Ecuador, 2019) refuerzan la capacidad de razonar, pensar, comunicar, aplicar y valorar las relaciones entre las ideas y los fenómenos reales, con apoyo en criterios de selección y jerarquización.

- La tercera etapa engloba actividades sincrónicas aplicadas durante el desarrollo de la clase, utilizando para fortalecer el interés de los educandos herramientas colaborativas como: Plataforma Microsoft Teams (clase virtual); el Software Matemático Interactivo Geogebra, que facilita la comprensión de los temas, pues, conlleva al trabajo autónomo, colaborativo e interactivo, generando actitudes de responsabilidad consigo mismo y con el entorno social.

- En la cuarta etapa, para comprobar la comprensión del conocimiento se establece una Evaluación, debiendo el estudiante acceder a través de la lectura de los Códigos QR, herramienta que proporciona una respuesta rápida a una determinada información que es ingresada y codificada con anterioridad. Cabe agregar, el docente crea el código QR dando la oportunidad a los educandos de retroalimentarse mediante la visualización de los recursos; en este sentido, Román \& Martín (2013) afirman que los estudiantes desarrollan habilidades, destrezas y competencias básicas de búsqueda de información en la red, edición gráfica mediante software específico, aspectos lúdicos, entre otros, mientras asimilan diferentes contenidos. 
Ejercicios resueltos. Consiste en el material disponible que puede ser descargado con el propósito que el educando refuerce su conocimiento sobre la temática de Cónicas. Además, se presenta el apartado "Tu criterio es importante", que implica una encuesta la cual tiene el propósito de recabar información de los usuarios que permita mejorar permanentemente el uso de la herramienta tecnológica.

Para cerrar, es importante resaltar que el uso de las TIC en los distintos ambientes educativos conlleva a mejorar la calidad y el nivel de competitividad del profesorado, por esta razón con el manejo de la estrategia tecnológica "Matematic Flipped Classroom" se han de desarrollar nuevas capacidades enfocadas en la apropiación pedagógica, la innovación en los materiales de aprendizaje y de formación constante en los procesos didácticos.

\section{Análisis, encuesta dirigida a los estudiantes, después de aplicar la estrategia tecnológica}

Una vez desarrollada la estrategia basada en las TIC para la mejora del desempeño académico en el área de Matemática, se aplicó un post test encontrándose que el 97\% de los estudiantes encuestados han fortalecido su aprendizaje a través de la simulación, observación y exploración, siendo capaces de resolver ejercicios matemáticos en un proceso colectivo y socializado, de construcción del conocimiento que estimula el estilo de enseñar y aprender.

En la dimensión aprender con ayuda de herramientas tecnológicas: un 93\% refuerza el cumplimiento de actividades y tareas, el $100 \%$ participa de forma creativa durante las clases de la asignatura, el $97 \%$ ha retroalimentado las habilidades en cálculo mental, mientras el $100 \%$ mejoró las destrezas con criterio de desempeño, el razonamiento matemático, el pensamiento lógico y la habilidad para resolver problemas de la vida cotidiana. 


\section{En la dimensión proceso de enseñanza aprendizaje mediante el uso de la} tecnología: se tiene que a través de la aplicación de la estrategia de matemática el 97\% ha mejorado la motivación, la autonomía, la auto reflexión y la participación; además muestran visualización y comprensión de relaciones existentes entre los conceptos; mientras un $100 \%$ demuestra interés en la investigación y un 100\% utiliza textos videos archivos y audiovisuales que facilitan el alcance de objetivos educativos.

\section{En referente a la implementación de la estrategia tecnológica "Matematic Flipped} Classroom" se puede observar que el $100 \%$ de los docentes consideran trascendental la utilización de las herramientas informáticas en el proceso de enseñanza aprendizaje.

De allí que se puede deducir que las estrategias didácticas basadas en las TIC orientan en el planteamiento de actividades, en la organización de las tareas. De esta forma se cumple con los principios didácticos de individualización, socialización, autonomía y creatividad. Es decir, de forma particular el estudiante adquiere sus propias comprensiones, razonamientos e interpretaciones, componentes que en todas las ciencias contribuyen con el desarrollo de la inteligencia, la personalidad y la práctica de los valores para alcanzar una formación de ciudadanos con compromiso en el crecimiento personal y colectivo. 


\section{Correlación}

\section{Tabla 5.}

Correlación.

\begin{tabular}{|c|c|c|c|c|c|c|}
\hline & & & Aprender & Matemática & Enseñar & Proceso \\
\hline \multirow{12}{*}{$\begin{array}{l}\text { Rho de } \\
\text { Spearman }\end{array}$} & \multirow{3}{*}{ Aprender } & $\begin{array}{l}\text { Coeficiente de } \\
\text { correlación }\end{array}$ & 1,000 &, $697^{\star \star}$ &, $700^{* *}$ &, $818^{* \star}$ \\
\hline & & Sig. (bilateral) & . & ,000 & ,000 & ,000 \\
\hline & & $\mathrm{N}$ & 30 & 30 & 30 & 30 \\
\hline & \multirow{3}{*}{ Matemática } & $\begin{array}{l}\text { Coeficiente de } \\
\text { correlación }\end{array}$ &, $697^{\star *}$ & 1,000 &, $633^{* *}$ &, $661^{\star \star}$ \\
\hline & & Sig. (bilateral) & ,000 & . & ,000 & ,000 \\
\hline & & $\mathrm{N}$ & 30 & 30 & 30 & 30 \\
\hline & \multirow{3}{*}{ Enseñar } & $\begin{array}{l}\text { Coeficiente de } \\
\text { correlación }\end{array}$ &, $700^{* \star}$ &, $633^{* *}$ & 1,000 &, $793^{* *}$ \\
\hline & & Sig. (bilateral) & ,000 & ,000 & . & ,000 \\
\hline & & $\mathrm{N}$ & 30 & 30 & 30 & 30 \\
\hline & \multirow{3}{*}{ Proceso } & $\begin{array}{l}\text { Coeficiente de } \\
\text { correlación }\end{array}$ &, $818^{* *}$ &, $661^{* *}$ &, $793^{\star *}$ & 1,000 \\
\hline & & Sig. (bilateral) & ,000 & ,000 & ,000 & . \\
\hline & & $\mathrm{N}$ & 30 & 30 & 30 & 30 \\
\hline
\end{tabular}

a. La correlación es significativa al nivel 0,01 (bilateral).

b. Intervalo de confianza al $95 \%$

Fuente: Encuesta aplicada a los estudiantes.

Con una población de 60 estudiantes, de los cuales 30 participan en el grupo de control, y 30 en el grupo experimental, se realiza la correlación entre el pre test y el post test; así, en el muestreo de error simple, el intervalo de confianza toma una equivalencia del $95 \%$, junto con una significación de 0,01 y 0,05. De igual forma, la correlación de Rho de Spearman se ubica entre los valores 0,697 y 0,818 cantidades mayores a cero. En concreto, el coeficiente rho de Spearman se muestra con un valor aproximado a 1, lo que significa que existe una correlación fuerte y positiva, validando la idea asumida como hipótesis, en relación a que la aplicación de la estrategia didáctica basada en TIC permite, 
a los estudiantes de Segundo Año de Bachillerato de la Unidad Educativa Vicente León, mejorar su desempeño académico en el área de Matemática y desarrollar las competencias de pensar y razonar, plantear y resolver problemas, construir modelos matemáticos $\mathrm{y}$, usar recursos y herramientas.

\section{DISCUSIÓN}

Considerando el criterio de los maestros en relación a los resultados obtenidos con la propuesta de intervención para mejorar el desempeño académico en la enseñanzaaprendizaje sobre el contenido matemático denominado Cónicas aplicada a los estudiantes del Segundo Año de Bachillerato de la Unidad Educativa Vicente León, se tiene que los recursos tecnológicos y las aplicaciones informáticas y los softwares, orientan en la generación de nuevos conocimientos, saberes y capacidades, al contener procesadores, hojas de cálculo, editores de imagen, entre otros elementos, que aplicados en el aula facilitan el proceso de enseñanza aprendizaje, ya que motivan y promueven la participación del educando. Argumento que es sustentado tanto por Pacheco (2015) cuando expresa que los docentes mediante estrategias y materiales refuerzan la labor pedagógica, mientras a los educandos les ayuda en la comprensión de los contenidos en este caso matemáticos (pág. 57); como por Huertas \& Pantoja (2016) cuando expresan que el uso de las tecnologías de la información y comunicación impacta en el desarrollo de competencias a través de actividades que conllevan al trabajo en equipo, de forma activa y cooperativa (pág. 237),

Asimismo, con los resultados puede observarse que con la utilización de estrategias tecnológicas los estudiantes en el área de matemática comprenden conceptos, elementos, leyes, propiedades que posteriormente aplican reflexivamente en cálculos y operaciones. Situación tal, se armoniza con lo expresado tanto por Astudillo (2016), en cuanto a que los recursos, materiales y herramientas digitales permiten la combinación de la información con los conocimientos interiorizados mediante procesos de reflexión, cosa que conlleva a la solución de situaciones complejas que requieren relacionar los 
saberes asimilados, los métodos y actividades aprendidas por el educando para plantear, interpretar y resolver problemas de la vida (pág. 112). Como por Alcibar, Monroy, \& Jiménez, (2018), en cuanto a que las estrategias y recursos tecnológicos dan respuesta a la necesidad de utilizar metodologías que contribuyan en el desarrollo de la competencia reflexiva en la disciplina de la matemática, que requiere la consolidación de conocimientos y el dominio de los procesos, para fortalecer las competencias de razonamiento, abstracción, análisis, sistematización y resolución de problemas (pág. 102).

Para concretar, se puede decir que la incorporación de herramientas virtuales en los diferentes en el proceso de enseñanza aprendizaje de la matemática contribuye en la transformación educativa y social, planteamiento que colinda con lo enunciado por Garcés \& Alcíva (2016), en relación a que el estudiante mediante el uso de aulas virtuales, blogs y programas digitales, adquiere destrezas que le permiten buscar verdad (justicia), actuar con ética, y ser responsable en el aula (pág. 172).

\section{CONCLUSIONES}

El estudio permite precisar que la utilización de recursos tecnológicos aplicados en la enseñanza y el aprendizaje de la matemática se constituyen en una estrategia educacional que motiva al educando en la adquisición de experiencias significativas a través de simulaciones y herramientas interactivas que conllevan a la construcción de su conocimiento.

Didácticamente las clases dirigidas por los maestros, al facilitar el acceso a los computadores e internet, orientan el cumplimiento de los principios y estándares para la educación matemática.

Es trascendental la utilización de los códigos QR, para potenciar el empleo de diversos recursos didácticos que favorecen en la competencia digital, la motivación, la creatividad y comunicación, para crear situaciones que activan la búsqueda de información; situaciones que contribuyen con el proceso de aprendizaje de la matemática, el 
planteamiento de problemas y la solución de dificultades con base en competencias matemáticas.

Finalmente se puede enunciar que la aplicación de la estrategia tecnológica "Matematic Flipped Classroom", ha generado oportunidades que mejoran el desempeño académico en la enseñanza-aprendizaje de la matemática, benefician el desarrollo de competencias a través de la utilización de las TIC; de igual manera, ha promovido la optimización y el aprovechamiento de diferentes herramientas y recursos en concordancia con las exigencias y la necesidad de los estudiantes.

\section{FINANCIAMIENTO}

No monetario.

\section{AGRADECIMIENTO}

A la Unidad Educativa Vicente León y a la Pontificia Universidad Católica del Ecuador; por permitir el desarrollo y fomento de la investigación.

\section{REFERENCIAS}

Albañil, L., \& Tique, L. (2015). Diseño de una estrategia didáctica para la construcción del aprendizaje matemático por medio de la argumentación a través del uso de las tic, en estudiantes del grado sexto de la Institución Educativa Instituto Técnico Industrial [Design of a didactic strategy for the construction of mathematical learning through argumentation through the use of ICT, in students of the sixth grade of the Educational Institution Instituto Técnico Industrial]. Villavicencio, España: Universidad de los Llanos. Obtenido de https://n9.cl/cg18

Alcibar, M., Monroy, A., \& Jiménez, M. (2018). Impacto y Aprovechamiento de las Tecnologías de la Información y las Comunicaciones en la Educación Superior [Impact and Use of Information and Communication Technologies in Higher Education]. Información tecnológica, 29(5), 101-110. https://dx.doi.org/10.4067/S0718-07642018000500101 
Astudillo, M. (2016). La configuración didáctica de las estrategias de enseñanza con Tecnologías de la Información y la Comunicación (TIC) en las prácticas pedagógicas de las ingenierías [The didactic configuration of teaching strategies with Information and Communication Technologies (ICT) in the pedagogical practices of engineering]. Revista Usal. Ediciones Universidad Salamanca, 109131. doi:10.14201/eks2016172109131

Cardozo, R., Duarte, J., \& Fernández, F. (2018). Estrategia didáctica, mediada por TIC para mejorar las competencias lectoescritoras en estudiantes de primero de primaria [Didactic strategy, mediated by ICT to improve reading and writing skills in first grade students]. Saber, Ciencias y Libertad, 235-247.

Cenich, G., Araujo, S., \& Santos, G. (30 de Abril de 2020). Conocimiento tecnológico pedagógico del contenido en la enseñanza de matemática en el ciclo superior de la escuela secundaria [Technological pedagogical knowledge of the content in the teaching of mathematics in the upper cycle of secondary school]. Perfiles educativos , 42(167), 15.

Domínguez, H., \& Carmona, H. (2017). El uso de las TIC y sus implicaciones en el rendimiento de los alumnos de bachillerato. Un primer acercamiento [The use of ICT and its implications on the performance of high school students. A first approach]. Education in the knowledge Society (EKS), 21-38. doi:10.14201/eks20171812138.

Faúndez, C., Bravo, A., Ramírez, G., \& Astudillo, H. (2017). Tecnologías de la Información y la Comunicación (TIC) en el Proceso de Enseñanza-Aprendizaje de Conceptos de Termodinámica como Herramienta para Futuros Docentes [Information and Communication Technologies (ICT) in the Teaching-Learning Process of Thermodynamic Concepts as a Tool for Future Teachers]. Formación Universitaria, 43-54. doi: $\underline{10.4067 / S 0718-50062017000400005}$

Fuentes, C., Paéz, O., \& Prieto, D. (2019). Dificultades de la resolución de problemas matemáticos de estudiantes de grado 501 Colegio Floresta Sur, sede b, jornada tarde, Localidad de Kennedy. Bogotá, D.C. [Difficulties in solving mathematical problems of students in grade 501 Floresta Sur College, headquarters b, afternoon session, Kennedy Town. Bogotá, D.C.], Colombia: Universidad Cooperativa de Colombia. https://n9.cl/2mm5 
Garcés, E., \& Alcívar, O. (2016). Las tecnologías de la información en el cambio de la eduación superior en el siglo XXI: reflexiones para la práctica [Information technologies in the change of higher education in the XXI century: reflections for practice]. Universidad y Sociedad, 171-177.

García, M., \& Benítez, A. (Abril de 2011). Competencias Matemáticas Desarrolladas en Ambientes Virtuales de Aprendizaje: el Caso de MOODLE [Mathematical Competences Developed in Virtual Learning Environments: the MOODLE Case]. Formación universitaria, 4(3), 31-42. https://dx.doi.org/10.4067/S0718$\underline{50062011000300005}$

Grisales, A. (Diciembre de 2018). Uso de recursos TIC en la enseñanza de las matemáticas: retos y perspectivas [Use of ICT resources in the teaching of mathematics: challenges and perspectives]. Entramado, 14(2), 198-214. https://n9.cl/cvin

Heredia, V. (14 de Marzo de 2021). Resultados de la evaluación PISA-D plantean varios retos en educación [Results of the PISA-D assessment pose several challenges in education]. Preuniversitario Newton, 7. https://n9.cl/iq0i5

Herrada, R., \& Baños, R. (10 de Septiembre de 2018). Experiencias de aprendizaje cooperativo en matemáticas. Espiral [Cooperative learning experiences in mathematics. Spiral]. Cuadernos del profesorado, 11(23), 10. https://n9.cl/02upg

Herrera, S., Novelo, S., Díaz, J., \& Hernández, H. (Junio de 2016). Estrategias de enseñanza para las matemáticas en el nivel superior [Teaching Strategies for Higher Level Mathematics]. Revista Iberoamericana de Producción Académica y Gestión Educativa , 3 (5).

Huertas, A., \& Pantoja, A. (2016). Efectos de un programa educativo basado en el uso de las TIC, sobre el rendimiento académico y la motivación del alumnado en la asignatura de tecnología de educación secundaria [Effects of an educational program based on the use of ICT, on the academic performance and motivation of students in the subject of secondary education technology]. Revista Educación $X X 1,229-250$.

Íñiguez, F. (15 de Marzo de 2015). El desarrollo de la competencia matemática en el aula de ciencias experimentales [The development of mathematical competence in the experimental science classroom]. Didáctica de las Ciencias y de la Matemática. 67(2), 14. https://n9.cl/fywgr 
Instituto Nacional de Evaluación Educativa. (2019). Informe de Resultados Ser Estudiante 2017-2018 [Results Report Being a Student 2017-2018]. Quito, Ecuador: Ministerio de Educación del Ecuador. Obtenido de https://n9.cl/mzcab

Khan, M., \& Markauskaite, L. (2017). Approaches to ICT-enhaced teaching in technical and vocational education: a phenomenographic . Higher Educa, 691-707. doi:10.1007/s10734-016-9990-2

Mainato, R., \& Drouet, M. (2017). La tecnología del aprendizaje y conocimiento en el desempeño académico de los estudiantes de noveno año de educación básica superior en la asignatura Matemática [The technology of learning and knowledge in the academic performance of students in the ninth year of higher basic education in the subject Mathematics]. Guayaquil, Ecuador: Universidad de Guayaquil. https://n9.cl/wijco

Martínez, L., \& Pérez, V. (Abril de 2016). El desarrollo de la Matemática y su relación con la tecnología y la sociedad [The development of Mathematics and its relationship with technology and society]. Universidad y Sociedad. Cienfuegos, 8(1), 9. http://scielo.sld.cu/pdf/rus/v8n1/rus14116.pdf

Matas, A. (Marzo de 2018). Diseño del formato de escalas tipo Likert: un estado de la cuestion [Design of the Likert-type scale format: a state of the art]. Revista electrónica de investigación educativa. REDIE , 20(1), 10. http://www.scielo.org.mx/pdf/redie/v20n1/1607-4041-redie-20-01-38.pdf

Mejía, G., Toala, G., \& Valverde, A. (2017). Modelo para evaluar el uso de la tecnología para el aprendizaje y la adquisición del conocimiento dentro del proceso de enseñanza aprendizaje [Model to evaluate the use of technology for learning and the acquisition of knowledge within the teaching-learning process]. Revista Publicando. Universidad Central del Ecuador, 4(11), 20. https://revistapublicando.org/revista/index.php/crv/article/view/523

Ministerio de Educación del Ecuador. (2019). Currículo de los Niveles de Educación Obligatoria. Nivel Bachillerato [Curriculum of the Compulsory Education Levels. Baccalaureate level] (Segunda Edición ed.) . Quito, Ecuador: Ministerio de Educación del Ecuador. https://n9.cl/1frin 
Niebles, W., Hernàndez, H., \& Cardona, D. (2016). Gestión tecnológica del conocimiento: herramienta moderna para la gerencia de instituciones educativas [Technological knowledge management: modern tool for the management of educational institutions]. Revista de investigación, desarrollo e innovación , 25-36.

Pacheco, F. (2015). Las TIC como herramientas en el proceso de enseñanza aprendizaje para optimizar el rendimiento académico [ICT as tools in the teachinglearning process to optimize academic performance]. Revista Ciencias Pedagógicas e Innovación UPSE, 56-62.

Palella, S. \& Martins, F. (2015). Metodología de la Investigación Cuantitativa [Quantitative Research Methodology]. Caracas. FEDUPEL.

Román, P., \& Martín, A. (Octubre de 2013). La formación de docentes en estrategias innovadoras de enseñanza y aprendizaje: los códigos de respuesta rápida o códigos $Q R[$ Teacher training in innovative teaching and learning strategies: quick response codes or QR codes]. Didáctica, Innovación y Multimedia (DIM), 14.

Tamayo, A., Oscar, E., Zona, R., Loaiza, Z., \& Yasaldez, E. (30 de Septiembre de 2015). El pensamiento crítico en la educación. Algunas categorías centrales en su estudio [Critical thinking in education. Some central categories in your study]. Revista Latinoamericana de Estudios Educativos (Colombia). Universidad de Caldas Manizales, 11(2), 111-133.

Urgilez, R., \& Valdez, C. (2020). Educarse en la era digital: hábitos y prácticas de uso de plataformas digitales y redes sociales para el aprendizaje de la Matemática en el Subnivel Básica Superior de la Unidad Educativa Luis Cordero [Getting educated in the digital age: habits and practices of using digital platforms and social networks for learning Mathematics in the Upper Basic Sublevel of the Luis Cordero Educational Unit] . Azogues, Ecuador: Universidad Nacional de Educación. http://repositorio.unae.edu.ec/handle/123456789/1414 